\title{
III. On the Australian Species of the Coleopterous Genus Bolboceras, Kirby.
}

By J. O. Westwood, Esq., F.L.S.

Read March 21st, 1848.

BOLBOCERAS, a genus of Lamellicorn beetles, was proposed by the Rev. W. Kirby, in his excellent memoir published thirty years ago, in the 12th volume of the 'Transactions of the Linnean Society,' containing descriptions of New Australian Coleoptera collected by Mr. Robert Brown. In his observations appended to the Latin characters given of the genus, as contrasted with those of Geotrupes, Mr. Kirby more especially endeavoured to point out the differences between these two genera, alluding, among other things, to the structure of the outer lobe of the maxillæ, but not mentioning (except in the Latin character) the horny inner lobe, with its bipartite structure, a character (as Mr. MacLeay has shown) of the highest importance, as distinctly indicating a variety in the operation of manducation, and consequently in the nature of the food of the insects. It is evident that Mr. Kirby's description of the maxilla of Bolboceras was drawn from the inaccurate figure of that part given in pl. 23. fig. $5 d$, which omits the upper portion of the lower lobe of the maxilla, which is thereby reduced to a single horny point. The maxilla of Bolboceras is, however, almost exactly similar to that of Athyreus; indicating, in conjunction with the general appearance of the insects, an analogous mode of life, although the difference in the place of insertion of the middle feet must evidently be regarded as a proof of some distinction in the habits of the two genera.

Mr. Kirby's observations, it will be further seen, are directed to the structure of the antennæ and trophi alone. There are consequently two important characters derived from the external structure of the other parts of the insects which separate the two genera, rendering Bolboceras also as far removed from the type of the family Geotrupida as Athyreus. These are, the entire structure of the eyes in Bolboceras, whilst each eye is divided into two parts by a distinct septum in Geotrupes, and the want of a patch of orange plush-like hairs on the thighs of the fore-legs, which exists in Geotrupes.

Mr. Kirby mentions as belonging to Bolboceras, "Sc. mobilicornis, Linn. (of which Sc. testaceus, Fabr., is only a variety), quadridens, Linn., farctus, Fabr., Lazarus, Fabr., Cyclops, Oliv., \&c.," together with the new species which he described under the name of Bolboceras australasice, a name now proved to be inapplicable, not only because we possess many Australian species of the genus, but also because the insect so described is but the female of another species. Of the above-mentioned species, the first, Sc. mobilicornis, does not belong to the genus Bolboceras, from which it is distinguished by its bipartite eyes, although the structure of the maxillæ and the want of a patch of plush on the fore femora show its near approach to it. It will therefore be proper to apply the generic name of Odontaus, given to this genus by Koppe, instead of Bolboceras, as it stands at 
present in English collections and books. The species, moreover, which it will be advisable to regard as the type of Bolboceras, will be Sc. quadridens, Linn., as that was the species dissected by Mr. Kirby.

In $1819 \mathrm{Mr}$. MacLeay published the description of his genus Elephastomus in the first part of his 'Horæ Entomologicæ,' founded upon the singular Australian Scarabaus proboscideus, first described by Schreibers in the 6th volume of the 'Linnean Transactions.' In this strange insect the crown of the head is extended forwards, so as to push the ordinary front part of the head, including the clypeus, mandibles and labrum, quite underneath it; just as if the human forehead were dilated over the entire face, and the nose and mouth pushed between the chin and the throat; with this difference, that in the insect the horizontal upper lip and mandibles become perpendicular, whilst the opposite would be the case in a human head so deformed. Referring again to the structure of the maxillæ, we find Mr. MacLeay's description of those of Elephastomus, "Maxillæ corneæ, arcuatæ, intùs dente acuto et ad apicem laciniâ obtusâ ciliis spinosulis armatæ," incorrect, omitting to notice the upper portion of the inner lobe of the maxillæ; whilst the figure given of it, pl. 2. fig. $10 \mathrm{E}$, is still more incorrect, omitting both the horny teeth of the lower lobe. In this, however, and all its essential characters, this insect approaches so closely to Bolboceras Australasia, that, long ago, I had attached to a figure of El.proboscideus the following note: "Is not Bolboceras australasia of Kirby the female? The box-like clava of their antennæ agrees;" and in Dr. Klug's excellent Monograph on the genera Athyreus and Bolboceras, published in the 'Transactions of the Berlin Academy' for 1843, we find Scarabaus proboscideus given as the first species of Bolboceras, with Bolb. Australasia as its female. In fact, with the exception of the extraordinary formation of the head, and the alteration consequent thereupon in the position of the trophi, we can find no variation in the structure of these organs of higher importance (with reference to the question of the retention of Elephastomus either as a genus or subgenus) than the greatly elongated maxillary palpi and the identical structure of the two mandibles, which are bifid at the tips.

The number of the species of the genus Bolboceras was considerably increased by the late Mr. Bainbridge, who published a short paper on some of the Australian species, from the Collection of the Rev. F. W. Hope, in the 'Transactions of the Entomological Society'; by Messrs. Guérin-Ménéville and Castelnau, in their several works; and especially by Dr. Klug, in the monograph above referred to.

As some confusion has been introduced into the nomenclature of the Australian species, and as I have to add several hitherto undescribed ones from New Holland to the list, including a closely allied new genus, I have thought it would be desirable in this paper to concentrate the whole of the New Holland Bolbocerata. It may be proper to add, that my quotations of the species described from the Collections of Messrs. Hope and Gory have been made from an examination of the type specimens in Mr. Hope's Collection, with which is now also incorporated that of M. Gory.

The circumstance of so many of the largest species of this genus being inhabitants of New Holland, where, in consequence of the absence of the larger mammalia, it is impossible for them to possess the same habit of burrowing into and under dung, as the Geotrupes of our moderate climates, is in itself a sufficient evidence that the Bolbocerata, like 
the Athyrei, must be regarded as very aberrant species in the family of which our Geotrupes is the well-known type.

It has been thought advisable that a continuation of this paper, containing descriptions of many additional new species of Bolboceras from India and other foreign regions, should be published as a distinct memoir, which accordingly appears as the following article.

\section{Bolboceras (Elephastomus) proboscideus, MacLeay, ut supr. citat.}

q Bolboceras Australasia, Kirby, loc. cit.

Obs. Caput et pronotum delineavi in TAB. III. fig. 1. ex individuo typico in Musæo Societatis Entomologicæ Londinensis.

Obs. 2. Figura maris in opere Griffith's Animal Kingdom, Ins. pl. 40. fig. 4, $4 a, 4 b$.

Obs. 3. Varietatem maris (cum fœeminâ Bolb. Australasice omninò congruenti copulâ captam) Societati Entomologicæ Londinensi communicavit D. F. Cox. Caput maris cornu perpendiculari infero caret, angulo parvo prominente locum ejus occupante.

2. Bolboceras (Elephastomus) Kirbir; castaneo-fulvus, capitis cornu antico porrecto brevi truncato plano subtùs in spinam bifidam haud producto, vertice carinâ brevi transversâ, prothoracis lateribus valdè punctatis utrinque fossulatis; disco posticè canali abbreviato longitudinali instructo.-Long. corp. lin. 9. (ТAB. III. fig. 2.)

Bolb. (Elephastomus) Kirbii, Hope MS.

Hab. in Terra Van Diemen. In Mus. Hope.

El. proboscideo minor, corpus castaneo-fulvum. Caput elongatum; yertice carinâ transversâ, quasi e tuberculis conjunctis formatâ, in medio instructo ; parte ante carinam porrectâ subquadratâ granulosâ anticè parum latiori apice utrinque rectè truncato, cornu antico abbreviato plano, anticè porrecto, apice truncato arcuato. Caput subtùs parte anticâ túmidâ; clypeo deflexo, labro ad apicem ejus affixo. Mandibulæ angustæ, fere similes, apice acuto denteque parvo subapicali armatæ. Prothorax anticè lævis, nitidus; lateribus punctatis et utrinque fossulâ rotundatâ instructus; disco ante medium carinâ tenui transversâ (in medio interruptâ) canalique tenui punctato longitudinali pone medium ante marginem posticum obliterato. Elytra striato-punctata; striis 14 in singulo elytro ad apicem elytrorum extensis. Tibiæ anticæ dentibus 6 nigris extùs armatæ.

Fig. $2 a$, caput et pronotum supra visa; $2 b$, caput a latere; $2 c$, labrum; $2 d$, mandibulæ; $2 e$, maxilla $*$; $2 f$, laciniæ labii cum palpis labialibus.

This appears to be the insect given by Mr. MacLeay as the female of Elephastomus proboscideus. It is however a male, and is given by Dr. Klug as a variety of the male of that species. The insect above described appears, however, to me to be sufficiently distinct as a species from the former + .

3. Bolboceras Reichir ; castaneus nitidus, capite cornu valdè elongato erecto, prothorace anticè valdè deflexo et subconcavo cornubus duobus crassis longitudine capitis porrectis lateralibus anticè armato: singulo versus basin dente obtuso erecto instructo; prothoracis lateribus rudè punctatis spatioque triangulari impresso et punctato ante scutellum; margine postico parùm elevato, elytris striis gracillimis punctatis, tibiis

* The upper division of the inner lobe is multispinose ; Dr. Klug has represented it as distinctly bifid in both sexes of B. Australasia.

+ The variety of the preceding species, wanting the perpendicular horn in front of the mouth, received since this sheet has been in type, seems to confirm Dr. Klug's opinion. 
anticis extùs 5-dentatis. $\delta^{7}$.--Long. corp. lin. 11; lat. prothoracis lin. 7. (ТАB. III. fig. 3.)

Hab. Port Essington. In Muss. Hope et Reiche.

б' Bolb. Reichii, Guérin, Voyage de la Favorite, p. 50, et Iconogr. du Règne Anim., Ins. p. 84.

Bolb. Kirbii ơ, Hope in Proc. Ent. Soc., Nov. 1841, p. 43.

\& Bolb. Kirbii, Bainbridge in Trans. Ent. Soc. vol. iii. p. 79.

Fœmina differt capite minori; vertice in tuberculum conicum apice bifidum elevato; clypeo et vertic

carinâ tenui angulatâ separatis ; pronoto anticè spatio subhexagono plano polito, in puncta duo profunda anticè lateraliter desinente, disco pone medium valdè punctato, versus marginem posticum elevato lævi, spatio ovali mediano punctato et impresso relicto.-Long. corp. lin. 11.

Hab. ad Melville Island. Mus. Hope (etiam in Mus. Gory, nunc Hope, cum nomine B. Reichii inscriptus). Fig. 3, mas magnit. naturalis ; $3 a$, caput suprà visum; $3 b$, caput et prothorax a latere visa ; $3 c$, mandibulæ maris ; $3 d$, caput et prothorax fœminæ suprà visa; $3 e$, eadem a latere visa.

Obs. The name given to the male of this species is here retained in preference to that of the female, in accordance with the usual custom in such cases.

4. Bolboceras serricollis, Bainbridge, Trans. Ent. Soc. vol. iii. p. 80. $\delta$.

Bolb. frontalis, Klug, Mon. Ath. et Bolb. p. 21. tab. 2. fig. $7 \& 7 a$. ( $\sigma^{\circ}$ 우)

\& Bolb. latus, Bainbridge, op. cit. vol. iii. part 2. p. 80 .

q Bolb. frontalis, Guérin, Ins. Voy. Favorite, Mag. Zool. 1838, p. 51 ; Klug, tab. supr. cit. fig. 7 b,c. Hab. ad Swan River. Mus. Hope.

5. Bolboceras hastifer, Bainbridge, op. cit. p. $81 . \delta^{\tau}$.

Athyreus recticornis, Guérin, Ins. Voy. Favorite, Mag. Zool. 1838, p. 49. pl. 232. fig. 1.

Bolb, recticornis, Klug, op. cit. p. 23. tab. 2. fig. 8.

Var. $\delta$ Bolb. fissicornis, Bainbridge, op. cit. p. 82.

Differt cornubus capitis et pronoti parùm brevioribus, hoc parùm crassiori et apice fisso.

Fig. $4 a$, caput et pronotum hujus varietatis maris suprà visa; $4 b$, eadem a latere; $4 c$, pars anterior capitis a latere; $4 d$, mandibulæ.

$H a b$. ad Swan River.

6. Bolboceras 3-tuberculatus, Bainbridge, op. cit. p. $82 . \delta^{\top}$.

Bolb. trituberculatus, Klug, op. cit. p. 24. no. 7 .

Bolb. thoracicornis, Gory, MS. in Mus.

Bolb. Guerini, Reiche, MS.

Fig. $5 a$, caput et prothorax maris suprà visa; $5 b$, mandibulæ maris; $5 c$, caput et prothorax fœminæ suprà visa; $5 d$, eadem a latere.

$H a b$. ad Swan River.

Obs. Mr. Bainbridge mistook the porrected closed mandibles of the male for the clypeus; his description, " clypeus quadridentatus medio emarginatus," being drawn from the mandibles. The female was sent me from Paris for examination by M. Reiche.

7. Bolboceras 7-tuberculatus, Bainbridge, op. cit. p. $81 . \delta^{7}$.

Bolb. excavatus, Klug, op. cit. p. 23. tab. 2. fig. 9. $\delta$.

$H a b$. ad Swan River.

Insectum hic describam, in Mus. Goryi olim conservatum, cum nomine manuscripto "Bolb. transversus, Gory" inscriptum. Hoc insectum Bolb. 7-tuberculati fœmineum existimavi, corpus totum fulvo- 
castaneum ; capite et pronoto punctatis; clypei margine antico recto simplici, posticè tuberculis $4 \mathrm{e}$ vertice separato, tuberculis duobus intermediis approximatis et paullò anterioribus, angulis lateralibus ante oculos acutis et parum reflexis. Pronotum impressione parvâ oblongâ in medio marginis antici ; disco transversim bicarinato, carinis lateraliter convergentibus spatium transversum subconcavum includentibus; impressione parvâ ovali nigricanti utrinque versus angulos posticos. Elytra lævia, striis gracillimis punctatis. (TAB. III. fig. $6 a$, caput et prothorax suprà visa; $6 b$, eadem a latere.)

Obs. Bolb. 7-tuberculatum fœminam Bolb. hastiferi esse autumat D. Reiche.

8. Bolboceras cononatus; " testaceus, capite punctato anticè medio 5- posticè utrinque 1-tuberculato, thorace rugoso, posticè transversim bicarinato dorso tuberculato, elytris punctato-striatis."-Long. lin. 9.

Bolb. coronatus, Klug, op. cit. p. 22. tab. 2. fig. 10, 10 a.

$H a b$. ad litt. occid. Novæ Hollandiæ. In Mus. D. Hope individuum pinguedine saturatum vidi.

9. BOLBOCERAS QUADRICORNIS ; " capite bicorni, thorace dorso cornubus duobus divaricatis armato lateribus profundè excavato, rufus, elytris nigris."-Long. corp. lin. $7 \frac{1}{2}$.

Bolb. quadricornis, Klug, op. cit. p. 22. tab. 2. fig. 6.

Hab. ad litt. occid. Novæ Hollandiæ; a me haud visus.

10. Bolboceras Taurus ; castaneus nitidus, capitis vertice utrinque laminis duabus auriculatis erectis instructo cornubusque duobus elongatis curvatis nigris ante oculos armato, pronoto in medio versus marginem anticum parùm reflexo seu tuberculis duobus transversis subelevatis instructo; lateribus punctatis.-Long. corp. lin. 8. (TAB. III. fig. 7.)

Bolb. Mimax, Reiche, MS.

Hab. ad Swan River. In Mus. Hope (olim Gory) sub nomine manuscripto hic conservato; etiam in Mus. Saunders.

Corpus suprà castaneum nitidum, pro latitudine parùm magis elongatum. Caput sub lente granulosum, tuberculis duobus erectis angulatis inter oculos ; verticis medio carinâ curvatâ parùm elevatâ utrinque in cornu porrectum curvatum nigrum (longitudine caput æquans) desinente. Clypeus declivis, granulosus. Mandibulæ parvæ, apice bidentatæ, dextræ margine externo magis rotundato, et ante apicem inciso. Antennæ, partes oris, et caput infrà fulvæ. Pronotum lateribus marginatis ciliatis et serrulatis, utrinque intra angulum anticum profundè impressum, anticè in medio versus marginem anticum glabrum et parùm elevatum, parte elevatâ in duas partes lineâ longitudinali impressâ divisâ, lateribus obliquè elevatis, rudè punctatis, et impressione rotundatâ parùm profundâ intra angulos posticos instructis. Elytra valdè convexa striis 7 punctatis ordinariis inter suturam et tubercula humeralia; lateribus etiam punctato-striatis. Scutellum læve. Pedes crassi. Tibiæ anticæ extùs dentibus 5 obtusis armatæ.

Fig. $7 a$, insectum magnitudine parùm auctum; $7 b$, caput et prothorax a latere visa; $7 c$, mandibulæ.

11. Bolboceras Capreolus; castaneus nitidus, capite posticè nigricanti; vertice cornu lato furcato 6-dentato erecto armato, pronoto anticè retuso glabro, dorso carinâ transversâ pone medium instructo, mandibulis magnis extùs denticulatis. $\delta$.-Long. corp. lin. 9. (ТАB. III. fig. 8.)

Bolb. diadematus, Reiche, MS.

Hab. in Novâ Hollandiâ, Swan River. In Mus. Hope (olim Mus. Gory), cum nomine suprà conservato designatum. Fœmina in Mus. D. Reiche. 
Corpus suprà castaneum nitidum. Caput labro porrecto, clypei margine antico tenui elevato in medio tuberculo minuto instructo ; frons glaberrima concava, suprà in medio verticis in cornu latum elevata, cujus apex anticè in dentes 4 acutos desinet, dentibusque duobus majoribus posticis versus pronotum inclinatis. Mandibulæ magnæ, porrectæ, concavæ, difformes; singula ante apicem externè in dentem porrectum subacutum elongata. Antennarum clava lutea. Prothorax elytris latior, anticè declivis nitidissimus et glaberrimus lineâque parùm impressâ longitudinali medianâ, carinâ transversâ utrinque abbreviatâ pone medium disci extensâ, pronoti parte posticâ lateribusque punctatis, his versus angulos posticos utrinque impressione ovali instructis. Elytra nitida tenuissimè punctatostriata, striis ante apicem desinentibus. Tibiæ anticæ 6-dentatæ, dentibus externis magnis acutis.

Fig. $8 a$, mas magnitudine parùm auctus; $8 b$, prothorax et caput a latere visa; $8 c$, mandibulæ maris; $8 d$, cornu verticale; $8 e$, prothorax et caput fœminæ a latere visa.

\section{Bolboceras neglectus, Hope, Proc. Ent. Soc. Nov. 1, 1841, p. 43.}

Fœmina sub hoc nomine in Mus. D. Hope hospitatur, Portûs Essingtonii, Novæ Hollandiæ, incola, quæ cum B. Reichii $q$ fere congruit, nisi magnitudine minori (lineas 9 tantùm longitudine habens) spatioque ovali depresso punctato ante scutellum carente; structurâ capitis, parteque politâ anticâ pronoti pone oculos in puncta duo magna profunda desinente, cum specie prædictâ convenit. An ejus varietas minor vel fœmina speciei alteræ?

Fig. $9 a$, caput et prothorax suprà visa.

13. Bolboceras BaINBrIDGII; piceus, capitis clypeo anticè tridentato : dente intermedio minori; vertice inermi, pronoto anticè valdè declivi dente erecto versus marginem anticum; parte declivi suprà carinâ cưrvatâ marginatâ.-Long. corp. lin. 7. (TAB. III. fig. 10.)

Hab. in Novâ Hollandiâ, ad Swan River. In Mus. D. Hope.

Præcedentibus minor. B. hastifero magis affinis, differt verò capite anticè tridentato. Caput suprà̀ planum; vertice fere lævi et inermi; clypeo anticè declivi suprà in lineam bisinuatam desinente, dentes tres efformante quorum laterales majores et magis porrecti. Mandibulæ difformes, dextra ante apicem externè lobo subrotundato instructa. Prothorax punctatus, punctis in medio disci magis remotis; parte anticâ valdè declivi, medio versus marginem anticum dente brevi erecto armatâ, et posticè lineâ curvatâ vel carinâ arcuatâ e parte posticâ separatâ: utrinque versus angulos posticos impressione rotundatâ instructus. Elytra punctato-striata, striis satis distinctis, sed ante apicem evanescentibus. Tibiæ anticæ extùs 6-dentatæ, dentibus duobus anticis (in specimine nostro unico) obliquè truncatis.

Fig. $10 a$, mas magnitudine parùm auctus; $10 b$, caput et prothorax e latere visa; $10 c$, mandibulæ.

Named in remembrance of the late Mr. W. Bainbridge, an assiduous collector of English insects, by whom descriptions of some Australian species of this genus were published in the 3rd volume of the Transactions of the Entomological Society of London.

14. Bolboceras rotundatus; parvus rotundatus rufo-castaneus glaberrimus, clypeo tuberculo elevato alteroque in medio verticis inter oculos, pronoto convexo vix punctato punctis paucis et valdè distantibus lineâque longitudinali medianâ impressâ punctatâ.-Long. corp. lin. 4. (ТАB. III. fig. 11.)

Bolb. rotundatus, Hope in Proc. Ent. Soc., Nov. 1, 1841, p. 43.

Hab. in Novâ Hollandiâ, ad Port Essington. In Mus. D. Hope.

Præcedentibus multò minor et ferè rotundatus. Corpus suprà glaberrimum convexum vix punctatum. Caput suprà in medio tuberculo (vel potius tuberculis duobus parvis conjunctis) instructum, margine 
antico in tuberculum elevato, lineis 4 elevatis ex hoc tuberculo prodeuntibus, scil. duabus ad angulos clypei anticos, duabusque ad basin antennarum; angulis 2 ante oculos rotundatis; parte capitis anteriori punctatâ. Mandibulæ difformes; dextra ut in multis aliis speciebus, lobo externo ante apicem instructa. Prothorax lineâ parùm impressâ longitudinali medianâ punctatâ, lateribus etiam versus angulos posticos impressionibus duabus rotundatis instructis. Elytra punctato-striata; striis ad apicem elytrorum extensis. Pedes antici longiores; tibiæ anticæ extùs 5-dentatæ.

Fig. $11 a$, caput et prothorax suprà visa; $11 b$, mandibulæ.

15. Bolboceras rubescens; fulvo-rufus glaberrimus, clypeo integro e vertice lineâ elevatâ separato, vertice tuberculis duobus minimis, prothorace convexo nitidissimo vix punctato; canali abbreviato in medio marginis antici punctisque nonnullis in lineam longitudinalem posticam dispositis fossulâque rotundatâ utrinque impressâ instructo. -Long. corp. ferè lin. 3. (ТАB. III. fig. 12.)

Bolb. rubescens, Hope, in Proc. Ent. Soc., Nov. 1, 1841, p. 43.

Hab. ad Port Essington Novæ Hollandiæ. In Mus. Hope.

B. rotundato valdè affinis; differt magnitudine minori, colore pallidiore, lineâque impressâ pronoti ferè obliteratâ.

Fig. $12 a$, caput et prothorax suprà visa.

16. Bolboceras corniculatus; rotundatus rufus glaberrimus, verticis margine antico ferè recto in medio subtuberculato; disco convexo utrinque inter oculos cornu erecto triangulari armato, pronoto sparsim punctato cornubus duobus contiguis versus marginem anticum tuberculisque duobus conicis lateralibus armato medioque fossulâ parvâ longitudinali, elytris striato-punctatis.-Long. corp. lin. 3. (TAB. III. fig. 13.) Bolb. corniculatus, Reiche, MSS.

Hab. ad Swan River Novæ Hollandiæ. In Mus. D. Reichii, Parisiis.

Parvus, rotundatus, rufus; elytris magis fulvis. Caput castaneo-rufum, suprà valdè concavum glaberrimum; verticis margine antico subrecto, tuberculo minuto medio instructo, ejusdem angulis anticis subacutis, utrinque etiam prope marginem oculorum internum spinâ conicâ erectâ armato. Antennæ fulvæ. Pronotum valdè convexum, glaberrimum, tenuissimè marginatum, punctis paucis notatum ; cornubus duobus contiguis apice obtusis prope marginem anticum, spatio transverso pone cornua impresso utrinque in tuberculum conicum desinente, dimidio postico, fossulâ tenui mediâ longitudinali punctatâ impresso, utrinque etiam versus marginem lateralem impressione parvâ rotundatâ punctatâ notato. Elytra glabra, valdè convexa; singulo striis decem punctorum, tribus externis cum tribus suturæ magis approximatis ad apicem conjunctis, intermediis abbreviatis. Pedes castaneorufi ; tibiæ anticæ extùs 6-dentatæ, dentibus obtusis, calcari anticarum elongato gracili, apice obtuso. An $B$. rotundati mas?

Fig. $13 a$, caput et prothorax suprà visa; $13 b$, eadem a latere visa.

\section{Stenaspipidus, Westw. Subgenus novum.}

Corpus magis elongatum quam in Bolboceratis veris; scutello elongato (nec triangulari); elytris striis tantùm quinque inter humeros et suturam; mesosterno porrecto. Differt etiam colore antennarum.

17. Bolboceras (Stenaspidius) Nigricornis; ovalis niger nitidus sparsim punctatus, capite tuberculo conico inter oculos, pronoto canali punctato medio aliisque duobus vOL. $\mathrm{XXI}$. 
abbreviatis pone oculos, elytris striato-punctatis.-Long. corp. lin. $3 \frac{1}{2}$. (TАB. III. fig. 14.)

Hab. in Novâ Hollandiâ. In Muss. D. Hope (olim Gory, cum nomine suprà indicato inscripto), Reiche et Westw.

Corpus suprà nigrum, nitidum. Caput mediocre. Clypeus e vertice lineâ elevatâ vel carinatâ in medio parùm angulatâ divisus, lineisque duabus brevioribus ad angulos posticos labri extensis. Antennæ nigræ; articulis parvis intermediis piceis. Mandibulæ ut in plurimis, dextra ante apicem externè lobo rotundato corneo instructa. Vertex tuberculo elevato inter oculos instructus; angulisque ante oculos rotundatis. Prothorax convexus, sparsim punctatus, lineâ longitudinali medianâ punctatâ impressâ alterisque duabus e margine antico pone oculos obliquè extensis at abbreviatis spatioque utrinque angusto parùm elevato, lævi, cum margine postico ferè parallelo, in fossulas 2 obliquas parùm profundas versus angulos posticos prothoracis desinente. Elytra striato-punctata, striis $\mathbf{5}$ inter humeros et suturam, aliisque 4 lateralibus. Tibiæ anticæ extùs 5-dentatæ. Scutellum elongatotriangulare. Mesosternum parùm angulato-porrectum.

Fig. $14 a$, insectum magnitudine auctum; $14 b$, caput, prothorax et mesosternum, a latere visa ; $14 c$, clypeus cum labro et mandibulis.

\section{EXPLANATION OF THE FIGURES.}

TAB. III.

Fig. 1. Bolboceras (Elephastomus) proboscideus, MacL.

Fig. 2. Bolboceras (Elephastomus) Kirbii, Hope.

Fig. 3. Bolboceras Reichii, Guér.

Fig. 4. Bolboceras hastifer, Bainbr. var.

Fig. 5. Bolboceras 3-tuberculatus, Bainbr.

Fig. 6. Bolboceras 7-tuberculatus, Bainbr. ㅇ?

Fig. 7. Bolboceras Taurus, Gory.

Fig. 8. Bolboceras Capreolus, Gory.

Fig. 9. Bolboceras neglectus, Hope.

Fig. 10. Bolboceras Bainbridgii, Westw.

Fig. 11. Bolboceras rotundatus, Hope.

Fig. 12. Bolboceras rubescens, Hope.

Fig. 13. Bolboceras corniculatus, Reiche.

Fig. 14. Bolboceras (Slenaspidius) nigricornis, Westw. 


\section{$2 \mathrm{BHL}$ Biodiversity Heritage Library}

Westwood, J. O. 1852. "On the Australian Species of the Coleopterous Genus Bolboceras, Kirby." Transactions of the Linnean Society of London 21, 11-18. https://doi.org/10.1111/j.1096-3642.1852.tb00495.x.

View This Item Online: https://www.biodiversitylibrary.org/item/19614

DOI: https://doi.org/10.1111/j.1096-3642.1852.tb00495.x

Permalink: https://www.biodiversitylibrary.org/partpdf/18212

\section{Holding Institution}

Natural History Museum Library, London

\section{Sponsored by}

Natural History Museum Library, London

\section{Copyright \& Reuse}

Copyright Status: Public domain. The BHL considers that this work is no longer under copyright protection.

This document was created from content at the Biodiversity Heritage Library, the world's largest open access digital library for biodiversity literature and archives. Visit BHL at https://www.biodiversitylibrary.org. 